\title{
TEMPO DE TELA, PERCEPÇÃO DA QUALIDADE DE SONO E EPISÓDIOS DE PARASSONIA EM ADOLESCENTES
}

SCREEN TIME, PERCEPTION OF SLEEP QUALITY AND EPISODES OF PARASOMNIA IN ADOLESCENTS

TIEMPO DE PANTALLA, PERCEPCIÓN DE LA CALIDAD DEL SUEÑO Y EPISODIOS DE PARASOMNIA EN ADOLESCENTES

Alison Oliveira da Silva' (Profissional de Educação Física)

Luciano Machado Ferreira Tenório de Oliveira ${ }^{2}$

(Profissional de Educação Física)

Marcos André Moura dos Santos (Profissional de Educação Física)

Rafael Miranda Tassitano 1,3

(Profissional de Educação Física)

1. Universidade de Pernambuco, Programa Associado de PósGraduação em Educação Física, Recife, PE, Brasil.

2. Universidade Federal de Pernambuco, Programa de PósGraduação em Neuropsiquiatria e Ciências do Comportamento, Recife, PE, Brasil.

3. Universidade Federal Rural de Pernambuco, Recife, PE, Brasil.

\section{Correspondência:}

Marcos André Moura dos Santos Escola Superior de Educação Física, Universidade de Pernambuco. Rua Arnóbio Marques, 310 , Santo Amaro, Recife, PE, Brasil. 50100-130.mmoura23@gmail.com

\section{RESUMO}

Introdução: O sono é um importante componente no processo de desenvolvimento biológico e mental das crianças e dos adolescentes, considerado fonte de revitalização das funções orgânicas. Objetivo: Analisar a associação entre o tipo e tempo de exposição à tela, a percepção da qualidade de sono e os episódios de parassonia em adolescentes. Métodos: Estudo transversal que incorpora um levantamento epidemiológico de base escolar com amostra representativa $(n=481)$ de estudantes (14 a 19 anos) do ensino médio da rede pública estadual do município de Caruaru, PE. Para a análise do sono e do estilo de vida, foi utilizada a versão traduzida e adaptada do Global School-Based Student Health Survey (GSHS). Recorreu-se à regressão logística binária para análise da associação entre as variáveis, considerando-se como desfecho a percepção negativa da qualidade de sono. Resultados: A prevalência de percepção negativa da qualidade de sono foi de 58\% (IC 95\% 53,5-62,3). Entre os comportamentos analisados, verificou-se que dormir oito horas ou menos por dia e assistir mais de duas horas de TV por dia aumentam, respectivamente, 2,69 (IC 95\% 1,61-4,71) e 1,71 (IC 95\% 1,08-2,73) as chances de relatar percepção negativa de sono. O tempo excessivo de tela, sobretudo diante da TV, esteve associado à maior quantidade de episódios de parassonia. Conclusão: A qualidade do sono está relacionada tanto com a quantidade de horas de sono, quanto com o tempo de exposição à TV. Além disso, uma quantidade maior de episódios de parassonia ocorreu entre os adolescentes que assistem mais de três horas de TV por dia.

Descritores: sono; televisão/utilização; estilo de vida sedentário.

\section{ABSTRACT}

Introduction: Sleep is an important component in the process of biological and mental development of children and adolescents, considered a source of revitalization of organic functions. Objective: To analyze the association between the screen type and time of exposure to the screen, the perception of sleep quality, and episodes of parasomnia in adolescents. Methods: A cross-sectional study that incorporates a school-based epidemiological survey with a representative sample ( $n=481$ ) of high school students (14-19years old) in the public education network of the city of Caruaru, PE. For the analysis of sleep and lifestyle, the translated and adapted version of the Global School-Based Student Health Survey (GSHS) was used. The binary logistic regression was used to analyze the association between the variables, considering the negative perception of sleep quality as an outcome. Results: The prevalence of negative perception of sleep quality was 58\% (95\% Cl 53.5-62.3). Among the behaviors analyzed, it was found that sleeping eight hours or less per day and watching television more than two hours per day increased, respectively, 2.69 (95\% Cl 1.61-4.71) and 1.71 (95\% Cl 1.08-2.73) the chances of reporting negative perception of sleep. Excessive screen time, especially in front of TV, was associated with a greater number of episodes of parasomnia. Conclusion: Sleep quality is related both to the number of hours of sleep and to the time of exposure to TV. In addition, a greater number of episodes of parasomnia occurred among adolescents who watched more than three hours of TV per day.

Keywords: sleep; television/utilization; sedentary lifestyle.

\section{RESUMEN}

Introducción: El sueño es un componente importante en el proceso de desarrollo biológico y mental de los niños y adolescentes y se considera una fuente de revitalización de las funciones orgánicas. Objetivo: Analizar la asociación entre el tipo y tiempo de exposición a la pantalla, la percepción de la calidad del sueño y los episodios de parasomnia en adolescentes. Métodos: Estudio transversal que incorpora una pesquisa epidemiológica de base escolar con muestra representativa $(n=481)$ de estudiantes (14 a 19 años) de la enseñanza media de la red pública estatal del municipio de Caruaru, PE. Para el análisis del sueño y del estilo de vida, se utilizó la versión traducida y adaptada del Global School-based Student Health Survey (GSHS). Se recurrió a la regresión logística binaria para analizar la asociación entre las variables, considerando como resultado la percepción negativa de la calidad del sueño. Resultados: La prevalencia de la percepción negativa de la calidad del sueño fue del 58\% (IC 95\%, 53,5-62,3). Entre los comportamientos analizados, se encontró que dormir ocho horas o menos al día y ver más de dos horas de TV al día aumentan, respectivamente, 2,69 (IC 95\%, 1,61-4,71) y 1,71 (IC 95\%, 1,08-2,73) las posibilidades de relatar la percepción negativa del sueño. El tiempo excesivo de pantalla, sobre todo ante la TV, se asoció con un mayor número 
de episodios de parasomnia. Conclusión: La calidad del sueño está relacionada tanto con la cantidad de horas de sueño, como con el tiempo de exposición a la TV. Además, una cantidad mayor de episodios de parasomnia ocurrió entre los adolescentes que asisten más de tres horas de televisión al día.

Descriptores: sueño; televisión/utilización; estilo de vida sedentario.

\section{INTRODUÇÃO}

O sono é um importante componente no processo de crescimento físico, desenvolvimento biológico e mental dos adolescentes ${ }^{1-3}$, considerado fonte de revitalização das funções orgânicas ${ }^{1}$ e associado a indicadores de saúde ${ }^{3}$. No Brasil, não se dispõe de dados nacionais para este subgrupo populacional, contudo em estudo realizado nas regiões Sul e Nordeste foi observado que 45,7\% dos adolescentes relataram percepção negativa da qualidade de sono ${ }^{4}$.

As alterações do padrão de sono na adolescência têm sido relatadas ao longo das últimas décadas ${ }^{5}$ e que podem ser explicadas, ao menos em parte, por alterações fisiológicas ${ }^{3}$ e por mudanças sociais e comportamentais ocorridas neste período ${ }^{1,2,6}$.

Relativamente, às mudanças comportamentais, estudos tem demostrado associação entre tempo e tipo de exposição à tela e percepção negativa da qualidade de sono ${ }^{6-12}$. Neste contexto, recentemente, uma revisão sistemática, reuniu 67 estudos com delineamentos transversais e longitudinais ${ }^{6}$. Foi observado em 90\% dos estudos a existência de associação significativa entre exposição ao tempo de tela e sono. O mecanismo subjacente que parece explicar estas associações aponta para a possibilidade da luz brilhante da tela suprimir a produção de melatonina, alterar aspectos do ciclo circadiano, aumentar a excitação mental e fisiológica ${ }^{13}$. Em adição evidências indicam que a exposição excessiva à tela causa menor tempo de duração ${ }^{7-10}$ e maior distúrbio de sono ${ }^{14}$, e que consequentemente uma percepção negativa da qualidade do sono ${ }^{4,11}$.

No entanto, os diferentes estudos investigaram unicamente a associação com a percepção da qualidade de sono, não sendo investigada a associação com as parassonias, conhecidas pela presença de manifestações motoras, autonômicas e psíquicas, que podem ocorrer no período de sono e são classificadas como distúrbios ${ }^{15}$. Neste contexto, até o momento, para o nosso conhecimento, é localizado na literatura um único estudo envolvendo crianças que abordou a associação entre distúrbios de sono e tempo de tela, especificamente televisão (TV) ${ }^{14}$. Deste modo, há uma lacuna de estudos que investigam o quanto da exposição elevada ao tempo de tela utilizando diferentes pontos de corte e diferentes tipos de exposição, estão associadas a esses distúrbios em adolescentes. Assim, o objetivo do estudo foi analisar a associação entre tempo e tipo de exposição de tela e a percepção da qualidade de sono e episódios de parassonias em adolescentes.

\section{MATERIAIS E MÉTODOS}

Estudo transversal de base escolar com abrangência municipal. A população alvo foi composta por adolescentes de ambos os gêneros, com idade entre 14 e 19 anos e regularmente matriculados no ensino médio da rede pública estadual de Caruaru/PE. Em 2013 um total de 8.833 estudantes, estavam matriculados e distribuídos em 15 escolas do município, segundo a Secretaria da Educação e Cultura do Estado (SEDUC).

Seguiram-se as determinações do Conselho Nacional de Saúde, sendo o projeto aprovado pelo Comitê de Ética em Pesquisa com seres humanos da faculdade ASCES (CAAE-22210913.8.0000.5203/CEP-ASCES: 072403/2013). A participação dos sujeitos foi voluntária e anônima, adotando-se a utilização de termo negativo de consentimento (parental passive consent form) para obter autorização dos pais de adolescentes com idade inferior a 18 anos, e termo de consentimento livre e esclarecido para obter anuência de participação daqueles estudantes com 18 e 19 anos de idade.

Para cálculo do tamanho amostral os seguintes parâmetros foram adotados: intervalo de confiança de 95\%; erro máximo tolerável de dois pontos percentuais; efeito do desenho amostral (deff) $=2$; e, por se tratar de um estudo abrangendo a análise de múltiplos comportamentos de risco e com diferentes frequências de ocorrência, utilizou-se prevalência de $50 \%$. A amostra inicial foi de 413 e adicionalmente, visando atenuar perdas e/ou preenchimento inadequado dos questionários, decidiu-se multiplicar o tamanho da amostra por 1,2 totalizando 491 estudantes.

Para seleção dos participantes do estudo, recorreu-se a uma amostragem por conglomerados em dois estágios, sendo que no primeiro estágio a escola foi a unidade amostral e no segundo estágio a turma. Todas as escolas foram consideradas elegíveis e o critério de seleção foi o porte (pequena $\leq 200$ estudantes matriculados; média $>200$ - 499 e grande $\geq 500$ ). Foram selecionadas nove escolas de forma proporcional ao porte, garantindo que pelo menos $50 \%$ das escolas de cada tamanho fossem selecionadas (2 escolas de grande porte, 4 de médio porte e 3 pequeno porte). Este procedimento foi adotado a fim de garantir que a amostra mantivesse a distribuição das escolas de acordo com as microrregiões do município.

No segundo estágio; considerou-se a quantidade de estudantes matriculados por turma nas escolas sorteadas como critério de seleção. Segundo dados da SEDUC cada turma tinha, em média, 39 alunos matriculados. Foram selecionadas proporcionalmente e aleatoriamente turmas em cada uma das nove escolas. Por fim, todos os estudantes das 20 turmas sorteadas foram convidados a participar, independentemente da idade. Todos os sorteios foram realizados pelo programa randomizer.

O instrumento utilizado foi o Global School-based Student Health Survey (GSHS), que foi validado e utilizado em outros estudos correlatos e no mesmo contexto do presente estudo. ${ }^{16,17}$ Foram utilizadas as sessões: Informações pessoais e da escola, atividade física, hábito alimentar e qualidade do sono, sendo acrescidas questões sobre a ocorrência de parassonias.

As informações sociodemográficas consideradas foram: gênero, idade (em anos), local de domicílio (rural ou urbano), renda familiar mensal, posse de aparelhos eletrônicos em casa (TV, Computador) e acesso a internet. Os fatores comportamentais foram: atividade física (quantidade de dias da semana que é fisicamente ativo por um total de pelo menos 60 minutos), fumo (sim ou não), álcool (sim ou não), consumo de frutas e de verduras (dias de consumo de frutas e verduras durante a semana). A percepção da qualidade de sono foi mensurada pela questão: "Com que frequência você considera que dorme bem?, com opções de resposta: sempre, a maioria das vezes, às vezes, quase nunca e nunca. A quantidade de horas dormidas foi mensurada a partir da questão: "Quantas horas você dorme, em média, durante a noite?", com opções de resposta em horas. Cinco episódios relacionados as parassonias foram levantados através das questões: "Alguém já falou que você costuma ranger os dentes durante o sono?",'Você costuma caminhar durante a noite enquanto dorme?", "Alguém já falou que você costuma falar enquanto dorme?", "Você costuma ter pesadelos uma ou mais vezes por semana?", "Você costuma gritar durante o sono e acordar apavorado(a) e amedrontado(a)? " com opções de resposta "Sim" e "Não". 
Tipo e tempo de exposição de tela foi mensurado a partir de duas questões, uma relacionada à TV: "Durante uma semana típica ou normal, quantas horas por dia você assiste TV?" e outra relacionada ao computador e/ou videogame: "Durante uma semana típica ou normal, quantas horas por dia você usa computador e/ou joga videogame?". Para ambas as questões a opção de resposta foi em horas.

A coleta dos dados ocorreu entre Junho e Novembro de 2014 e foi realizada por uma equipe de pesquisadores previamente capacitada. A aplicação dos questionários foi efetuada em sala de aula, na forma de entrevista coletiva, sem a presença dos professores e os estudantes tiveram oportunidade de esclarecer as dúvidas durante o preenchimento dos questionários (duração média: 30 e 40 minutos). Os dados foram tabulados no programa EpiData versão 3.1 (Epidata Association, Odense, Dinamarca) e foram realizados os procedimentos de controle de entrada de dados por meio da função check (controles). A fim de detectar possíveis erros, a entrada de dados foi repetida e por meio da função de comparação de arquivos duplicados. Os erros de digitação foram detectados e corrigidos.

A análise dos dados foi realizada no programa SPSS 17.0. Inicialmente foi realizada análise descritiva de todas as variáveis. Na análise inferencial, algumas variáveis foram dicotomizadas: atividade física ( $\geq 300$ minutos/ semana; < 300 minutos/ semana), fumo (Sim; Não), álcool (Sim; Não), consumo de frutas ( $\geq 5$ dias por semana; $<5$ dias por semana) e de verduras ( $\geq 5$ dias por semana; $<5$ dias por semana), renda familiar (até $R \$$ 1.000; maior que R\$ 1.000,00), trabalho (Sim; Não) e local de residência (Urbano; Rural). A percepção da qualidade de sono foi dicotomizada em positiva (sempre e na maioria das vezes) e negativa (às vezes, quase nunca e nunca) $)^{6}$. Quantidade de horas de sono durante a noite foi dicotomizada em exposto ( $<8$ horas/noite) e não exposto ( $\geq 8$ horas/noite) $)^{6,8}$.

As questões sobre parassonias foram agrupadas a partir do somatório das respostas positivas (presença): nenhum episódio, um episódio, dois episódios, três ou mais episódios. Para exposição ao tempo de tela foi utilizado $\theta$ ponto de corte $<2$ horas/dia (não expostos) e $\geq 2$ horas/ dia (expostos) 6,8 . Adicionalmente, ponto de corte $>3$ horas/dia de exposição à tela, TV e computador foi utilizado para analisar associação aos episódios de parassonias. Na análise de regressão logística binária foram feitas análises bruta e posteriormente ajustada para todas as variáveis, considerando a percepção negativa da qualidade de sono como variável desfecho.

\section{RESULTADOS}

Um total 569 estudantes (20 turmas) das nove escolas estavam presentes no dia da visita e foram entrevistados. Destes, 57 se recusaram a participar do estudo (10,1\%), totalizando 512 estudantes. A amostra final foi composta por estudantes com idade entre 14 e 19 anos, totalizando 481. A maioria dos estudantes eram moças (54,1\%), com até 16 anos (65,4\%), residentes da zona urbana $(84,6 \%)$ e não trabalhadores $(83,4 \%)$. As características sociais, demográficas e econômicas; estratificado por gênero estão na Tabela 1.

As prevalências de percepção negativa da qualidade de sono e tempo insuficiente de sono foram 58\% (IC95\% 53,5 - 62,3) e 77,1\% (IC95\% 72,9 - 80,4), respectivamente. Na análise bruta a percepção negativa da qualidade de sono esteve significativamente associada ao tempo insuficiente de sono (RP=2,69; IC95\% 1,53 - 4,69), ao tempo excessivo de TV $(R P=1,49 ;$ IC95\% 1,03 - 2,16) e ao consumo $<5$ dias/semana de frutas $(R P=1,48 ; I C 95 \% 1,63-2,13)$. Quando ajustada para as demais variáveis de confusão, o tempo de sono $(R P=2,75$; IC $95 \% 1,61-4,71)$ e o tempo excessivo de TV $(\mathrm{RP}=1,71 ; \mathrm{IC} 95 \% 1,08-2,73)$ permaneceram significativos. Verificou-se que dormir $<8$ horas/dia e assistir $\geq 2$ horas/dia de TV aumenta, respectivamente, 2,69 (IC95\%1,61 - 4,71) e 1,71 (IC95\% $1,08-2,73)$ a chance de relatar percepção negativa de sono (Tabela 2).
Tabela 1. Características socioeconômicas, demográficas de estudantes do Ensino Médio da Rede Pública Estadual de Caruaru, Pernambuco, Brasil, 2014.

\begin{tabular}{|c|c|c|c|c|c|c|c|}
\hline \multirow{2}{*}{ Variáveis } & \multicolumn{2}{|c|}{ Rapazes } & \multicolumn{2}{|c|}{ Moças } & \multicolumn{2}{|c|}{ Todos } & \multirow{2}{*}{$p$} \\
\hline & $\mathrm{N}$ & $\%$ & $\mathrm{~N}$ & $\%$ & $\mathrm{~N}$ & $\%$ & \\
\hline \multicolumn{7}{|l|}{ Idade (anos) } & \multirow{7}{*}{0,10} \\
\hline 14 & 17 & 7,9 & 37 & 14,2 & 54 & 11,2 & \\
\hline 15 & 56 & 25,3 & 74 & 28,5 & 130 & 27,0 & \\
\hline 16 & 53 & 24,0 & 59 & 22,7 & 112 & 23,3 & \\
\hline 17 & 58 & 26,2 & 49 & 18,8 & 107 & 22,2 & \\
\hline 18 & 25 & 11,3 & 31 & 11,9 & 56 & 11,6 & \\
\hline 19 & 12 & 5,4 & 10 & 3,8 & 22 & 4,6 & \\
\hline \multicolumn{7}{|l|}{ Cor da pele } & \multirow{6}{*}{0,11} \\
\hline Branca & 61 & 27,7 & 67 & 25,9 & 128 & 26,7 & \\
\hline Preta & 19 & 8,6 & 12 & 4,6 & 31 & 6,5 & \\
\hline Parda & 118 & 53,6 & 136 & 52,5 & 254 & 53,0 & \\
\hline Amarela & 14 & 6,4 & 30 & 11,6 & 44 & 9,2 & \\
\hline Indígena & 8 & 3,6 & 14 & 5,4 & 22 & 4,6 & \\
\hline \multicolumn{7}{|l|}{ Local de residência } & \multirow{3}{*}{1,00} \\
\hline Urbano & 187 & 84,6 & 220 & 84,6 & 407 & 84,6 & \\
\hline Rural & 34 & 15,4 & 40 & 15,4 & 74 & 15,4 & \\
\hline \multicolumn{7}{|l|}{ Trabalho } & \multirow{4}{*}{$<0,001$} \\
\hline Não & 141 & 64,1 & 216 & 83,4 & 357 & 74,5 & \\
\hline Até 20 horas semanais & 43 & 19,5 & 27 & 10,4 & 70 & 14,6 & \\
\hline Mais de 20 horas semanais & 36 & 16,4 & 16 & 6,2 & 52 & 10,9 & \\
\hline \multicolumn{7}{|l|}{ Escolaridade da mãe } & \multirow{4}{*}{0,70} \\
\hline Nunca estudou & 11 & 5,0 & 17 & 6,6 & 28 & 5,8 & \\
\hline Até 8 anos de estudo & 110 & 50,0 & 132 & 51,0 & 242 & 50,5 & \\
\hline Mais de 8 anos de estudo & 99 & 45,0 & 110 & 42,4 & 209 & 43,6 & \\
\hline \multicolumn{7}{|l|}{ Renda familiar mensal } & \multirow{3}{*}{0,09} \\
\hline Até $R \$ 1.000$ & 115 & 53,0 & 157 & 60,6 & 272 & 57,1 & \\
\hline Maior que $\mathrm{R} \$ 1.000$ & 102 & 47,0 & 102 & 39,4 & 204 & 42,9 & \\
\hline \multicolumn{7}{|l|}{ Tem televisão em casa? } & \multirow{3}{*}{0,19} \\
\hline Sim & 221 & 100 & 257 & 99,2 & 478 & 99,6 & \\
\hline Não & 0 & 0 & 2 & 0,8 & 2 & 0,4 & \\
\hline \multicolumn{7}{|l|}{ Tem computador em casa? } & \multirow{3}{*}{0,71} \\
\hline Sim & 164 & 74,5 & 190 & 73,1 & 354 & 73,8 & \\
\hline Não & 56 & 25,5 & 70 & 26,9 & 126 & 26,2 & \\
\hline \multicolumn{7}{|l|}{ Tem internet em casa? } & \multirow{3}{*}{0,49} \\
\hline Sim & 151 & 68,3 & 170 & 65,4 & 321 & 66,7 & \\
\hline Não & 70 & 31,7 & 90 & 34,6 & 160 & 33,3 & \\
\hline
\end{tabular}

Com relação aos episódios de parassonias, 88\% (IC95\% 84,9-90,7) dos adolescentes relataram ranger os dentes durante a noite, 10,8\% (IC95\% 8,5 - 14,1) caminhar enquanto dormem, 47,2\% (IC95\% 42,7 - 51,6) falar enquanto dormem, 34,1\% (IC95\% 30,0 - 38,4) ter pesadelos uma ou mais vezes por semana e 12,7\% (IC95\% 10,3 - 16,4) acordar amedrontado e/ou apavorado. A prevalência de adolescentes que não apresentaram nenhum episódio de parassonias foi de 32,9\% (IC95\% 28,8 - 37,1). Na Figura 1 está apresentada a associação entre os episódios de parassonias e a exposição ao tempo de tela com diferentes pontos de corte e considerando a exposição à TV e computador.

A Figura 2 apresenta a associação encontrada entre os episódios de parassonias e a exposição ao tempo de TV com diferentes pontos de corte. Foi identificado que a maior exposição ao tempo de TV foi associado positivamente com a ocorrência de parassonias.

Não foi observada nenhuma associação significativa entre os episódios de parassonias e o tempo de tela do computador, conforme apresentado na Figura 3.

\section{DISCUSSÃO}

Os resultados encontrados no estudo demostraram a existência de associação significativa entre tempo insuficiente de sono, tempo excessivo de TV e percepção negativa da qualidade do sono, mesmo após ajustes para fatores de confusão. Além disto, as parassonias estão associadas ao tempo excessivo de TV. Os estudantes que relataram > 3 horas/dia de tela, principalmente TV, apresentaram maior quantidade de episódios de parassonias em comparação as demais categorias de análise. 
Tabela 2. Razão de prevalência bruta e ajustada da percepção negativa da qualidade de sono de estudantes do Ensino Médio da Rede Pública Estadual de Caruaru, Pernambuco, Brasil, 2014.

\begin{tabular}{|c|c|c|c|c|c|c|c|}
\hline \multirow{2}{*}{ Variáveis } & \multirow{2}{*}{$\begin{array}{c}\text { Prevalên- } \\
\text { cia }\end{array}$} & \multicolumn{3}{|c|}{ Análise bruta } & \multicolumn{3}{|c|}{ Análise ajustada } \\
\hline & & $\mathrm{RP}$ & (IC)95\% & $p$ & $\mathrm{RP}$ & (IC)95\% & $p$ \\
\hline \multicolumn{8}{|l|}{ Gênero } \\
\hline Masculino & $134(60,6)$ & 1,00 & \multirow{2}{*}{$0,57-1,18$} & \multirow{2}{*}{0,28} & 1,00 & \multirow{2}{*}{$0,49-1,10$} & \multirow{2}{*}{0,13} \\
\hline Feminino & $145(55,8)$ & 0,82 & & & 0,73 & & \\
\hline \multicolumn{8}{|l|}{ Faixa etária } \\
\hline $14-16$ & $177(59,8)$ & 1,00 & \multirow{2}{*}{$0,57-1,20$} & \multirow{2}{*}{0,31} & 1,00 & \multirow{2}{*}{$0,57-1,27$} & \multirow{2}{*}{0,42} \\
\hline $17-19$ & $102(55,1)$ & 0,83 & & & 0,86 & & \\
\hline \multicolumn{8}{|l|}{ Local de residência } \\
\hline Urbano & $231(56,8)$ & 1,00 & \multirow{2}{*}{$0,84-2,36$} & \multirow{2}{*}{0,20} & 1,00 & \multirow{2}{*}{$0,81-2,17$} & \multirow{2}{*}{0,17} \\
\hline Rural & $48(64,9)$ & 1,40 & & & 1,34 & & \\
\hline \multicolumn{8}{|l|}{ Renda familiar } \\
\hline Até $R \$ 1.000$ & $158(58,1)$ & 1,00 & \multirow{2}{*}{$0,66-1,37$} & & 1,00 & \multirow{2}{*}{$0,65-1,41$} & > 01 \\
\hline Maior que $\mathrm{R} \$ 1.000$ & $116(56,9)$ & 0,95 & & 0,78 & 0,96 & & $0,1 / 1$ \\
\hline Trabalho & & & & & & & \\
\hline Não & $206(57,7)$ & 1,00 & $067-154$ & ר90 & 1,00 & $065-160$ & 086 \\
\hline Sim & $71(58,2)$ & 1,02 & $0,0 /-1,54$ & 0,92 & 1,04 & $0,00-1,09$ & 0,00 \\
\hline TV & & & & & & & \\
\hline$<2$ horas/dia & $94(51,9)$ & 1,00 & 102 & בחمि & 1,00 & ברד & ר רחת \\
\hline$\geq 2$ horas/dia & $185(61,7)$ & 1,49 & $1,03-2,10$ & 0,03 & 1,71 & $1,08-2,13$ & 0,02 \\
\hline CPU & & & & & & & \\
\hline$<2$ horas/dia & $137(59,8)$ & 1,00 & & 041 & 1,00 & & 071 \\
\hline$\geq 2$ horas/dia & $142(56,3)$ & 0,87 & $0,00-1,20$ & 0,44 & 0,91 & $|0,34-1,3|$ & 0,11 \\
\hline CPU com internet & & & & & & & \\
\hline$<2$ horas/dia & $88(27,4)$ & 1,00 & 187 & 021 & 1,00 & 107 & 016 \\
\hline$\geq 2$ horas/dia & $51(31,9)$ & 1,24 & $1,8 /$ & 0,31 & 1,34 & $0,91-1,91$ & 0,10 \\
\hline Tempo de tela & & & & & & & \\
\hline$<2$ horas/dia & $95(58,6)$ & 1,00 & $066-142$ & 087 & 1,00 & $047-160$ & 086 \\
\hline$\geq 2$ horas/dia & $183(57,9)$ & 0,97 & $0,00-1,42$ & $0,0 /$ & 0,85 & $0,47-1,09$ & 0,00 \\
\hline Atividade física & & & & & & & \\
\hline$\geq 300 \mathrm{~min} / \mathrm{semana}$ & $53(65,4)$ & 1,00 & & 012 & 1,00 & & 018 \\
\hline$<300 \mathrm{~min} / \mathrm{semana}$ & $223(56,3)$ & 0,68 & $0,41-1,12$ & 0,13 & 0,70 & $0,41-1,19$ & 0,18 \\
\hline Fumo & & & & & & & \\
\hline Não & $268(58,6)$ & 1,00 & & 021 & 1,00 & & 052 \\
\hline $\operatorname{Sim}$ & $11(45,8)$ & 0,60 & 1,50 & $0, \angle 1$ & 0,75 & $0,50-1,04$ & 0,32 \\
\hline Consumo de álcool & & & & & & & \\
\hline Não & $227(60,2)$ & 1,00 & & 807 & 1,00 & 107 & OمO \\
\hline Sim & $52(50,5)$ & 0,67 & 1,05 & 0,07 & 0,65 & $0,40-1,0]$ & 0,09 \\
\hline Consumo de fruta & & & & & & & \\
\hline$\geq 5$ dias na semana & $136(53,5)$ & 1,00 & $103-213$ & 003 & 1,00 & $292-201$ & 012 \\
\hline$<5$ dias na semana & $143(63,0)$ & 1,48 & 2,13 & 0,03 & 1,36 & $|0,92-2,0|$ & 0,12 \\
\hline Consumo de verdura & & & & & & & \\
\hline$\geq 5$ dias na semana & $185(55,9)$ & 1,00 & & 016 & 1,00 & & בح 0 \\
\hline$<5$ dias na semana & $94(62,7)$ & 1,32 & & 0,10 & 1,30 & $0,05-2,02$ & $0, \angle 2$ \\
\hline Tempo de sono & & & & & & & \\
\hline$\geq 8$ horas & $17(15,5)$ & 1,00 & 469 & 001 & 1,00 & & O \\
\hline$<8$ horas & $32,9(122)$ & 2,69 & $1,53-4,09$ & 0,01 & 2,75 & 1,01 & 0,01 \\
\hline
\end{tabular}

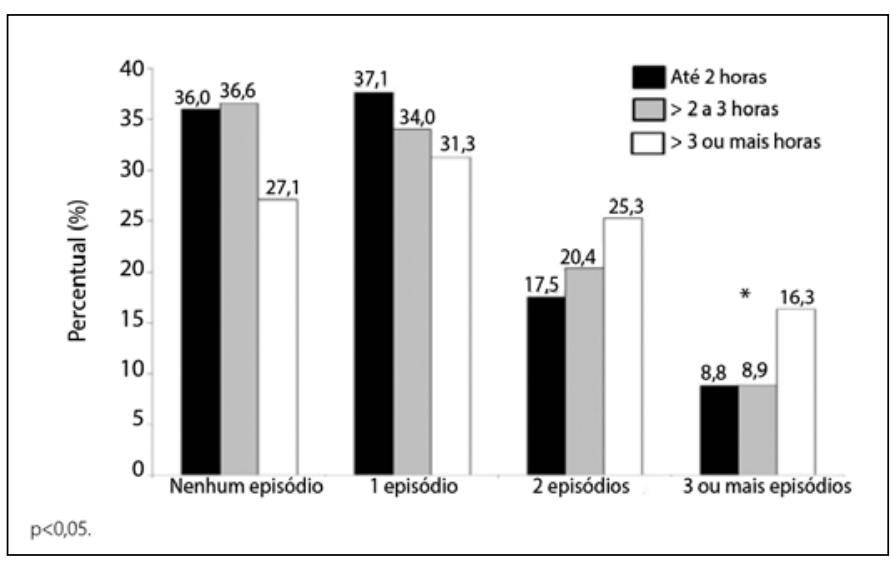

Figura 1. Comparação dentre as categorias de episódios de parassonias e tempo de exposição ao tempo de tela total.

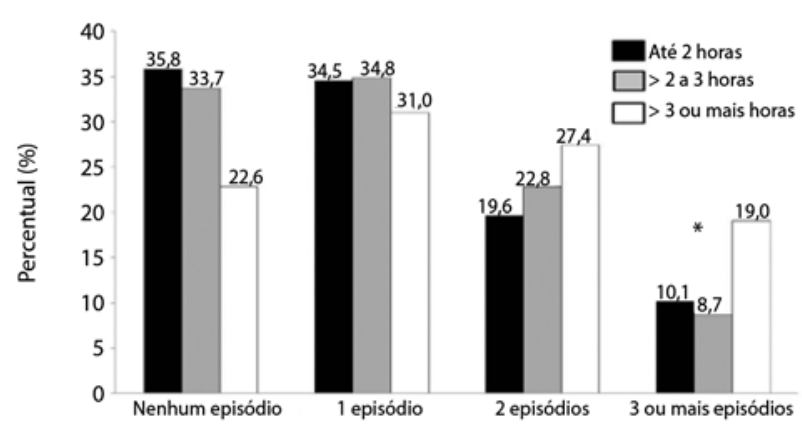

${ }^{*} \mathrm{p}<0,05$.

Figura 2. Comparação dentre as categorias de episódios de parassonias e tempo de exposição ao tempo de TV.

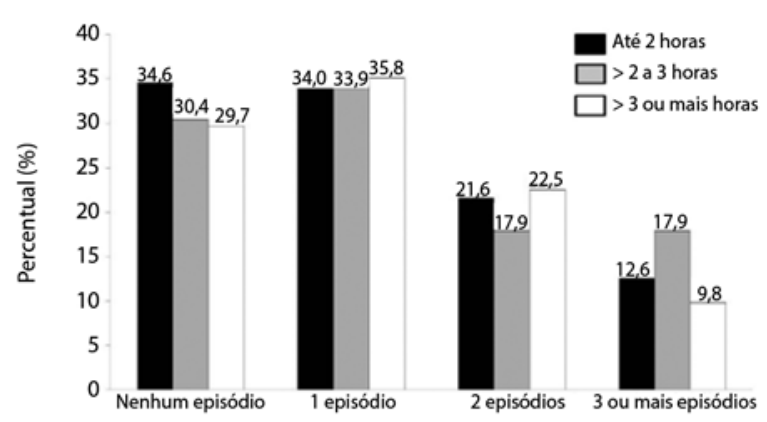

Figura 3. Comparação dentre as categorias de episódios de parassonias e tempo de exposição a computador.

Estudos têm demonstrado que as mudanças nos fatores ambientais $^{1,2}$, sociais ${ }^{18,19}$ e comportamentais ${ }^{2,6}$ parecem ser determinantes na alteração do padrão de sono, seja em quantidade como na qualidade. Estas evidências parecem plausíveis, contudo poucos estudos nacionais sobre a temática envolvendo adolescentes foram publicados e nenhum considerou cidade do interior.

Tem sido descrito que uma alta prevalência de sono insuficiente e de má qualidade entre os jovens $\mathbf{5}^{5,20}$ pode estar relacionado a determinados fatores biológicos e maturacionais, mudanças comportamentais e sociais, especificamente o aumento das obrigações escolares, atividades sociais ${ }^{2}$ e ao uso excessivo de equipamentos eletrônicos ${ }^{21}$. Neste contexto, os estudos de Hoefelmann et al..$^{18}$ e Bernardo et al. ${ }^{19}$ indicaram que a renda familiar, local de residência e o trabalho aumentam significativamente a percepção negativa do sono. Por exemplo, entre 2001 e 2011, foi observado um aumento de $31,2 \%$ na prevalência da percepção negativa da qualidade do sono entre os adolescentes catarinenses ${ }^{18}$. Os autores sugerem que este aumento pode ser explicado pelas mudanças nos fatores sociodemográficos, que por sua vez foi influenciado pela evolução tecnológica observada durante esta década. Contudo, no presente estudo nenhuma variável sociodemográfica esteve associada significativamente com o desfecho analisado.

Por outro lado, o tempo excessivo de TV ( $\geq 2$ horas/dia) esteve associado com a percepção negativa da qualidade do sono. Esta relação tem sido observada tanto em estudos transversais ${ }^{12}$, quanto em estudos longitudinais ${ }^{8}$. Esperava-se que o tempo excessivo no uso do computador também estivesse associado, conforme a literatura tem reportado 10,11. No entanto, acredita-se que este resultado pode estar relacionado ao acesso mais recente ao computador com internet em relação à TV, o que influencia os padrões de uso e a interatividade com os aparelhos ${ }^{22}$.

Entre os estudantes de Caruaru, apenas dois $(0,4 \%)$ relataram não possuir TV, enquanto que um em cada três estudantes não tem computador e um em cada quatro não tem acesso à internet em casa. 
Relatório do IBGE 22 (2013) indica que para algumas tecnologias, como a TV, o acesso já é quase total e não difere das cidades de grande porte, porém o computador com acesso a internet ainda é caro e, portanto, com menor acesso para parte da população. Acreditamos que o acesso consolidado, principalmente nas duas últimas décadas, estabeleceu hábitos regulares de utilização em relação à TV, o que ainda não acontece com o computador e podem explicar parcialmente os resultados.

O tempo insuficiente de sono (< oito horas) também esteve associado ao desfecho analisado. A diminuição do tempo de sono tem se tornado um problema na sociedade moderna, com o consequente aumento da sonolência diurna ${ }^{19}$, problemas de saúde mental ${ }^{12}$ e menor qualidade do sono ${ }^{23}$. As recomendações sobre a quantidade de horas de sono por noite para adolescentes foram modificadas ao longo da história. Uma revisão sistemática com dados seculares (1897 - 2009) indicou que o tempo de sono passou de nove e meio a dez horas de sono por noite, em 1910, para oito a nove horas em $2009^{5}$.

Um recente relatório da National Sleep Foundation indica que 60\% dos adolescentes americanos dormem menos de oito horas por noite ${ }^{24}$. No Brasil, não se dispõe de dados nacionais, contudo estudo realizado em uma capital da região Sul e uma capital da região Nordeste indicou que $76,7 \%$ dos adolescentes dormem menos que oito horas por noite ${ }^{4}$, proporção semelhante aos resultados encontrados no presente estudo. Parte da diminuição no tempo de sono suficiente pode está relacionada às mudanças nos padrões de sono na adolescência², ao aumento das obrigações escolares e das atividades sociais, além da exposição elevada ao tempo de tela ${ }^{6}$. Este novo padrão pode colaborar para que os adolescentes deitem mais tarde e acordem mais cedo, aumentando a sonolência diurna ${ }^{19}$ além dos possíveis episódios de parassonias ${ }^{15}$, contribuindo para uma menor qualidade do sono. Além disto, têm sido relatadas alterações no ciclo circadiano, particularmente as desordens na fase tardia do sono (DFTS) ${ }^{25}$, descrito como um problema comum entre os adolescentes após o início da puberdade. Estimativas sugerem que entre $5 \%$ e 10\% dos adolescentes podem apresentar DFTS, que pode ocorrer como resultado de fatores biológicos subjacentes, bem como as demandas ambientais e o estilo de vida ${ }^{26}$.

Uma vez que a percepção de qualidade do sono sofre influência da percepção subjetiva da pessoa, as parassonias podem ser afetadas por esta percepção, pois são episódios pontuais que interferem na qualidade do sono ${ }^{15}$. No presente estudo os adolescentes expostos há um tempo elevado de tela ( $>3$ horas/dia) apresentaram uma maior quantidade de episódios de parassonias. Quando analisado essa exposição excessiva por tipo de aparelho observou-se que apenas a exposição à TV esteve associada a um maior número de episódios de parassonias. Este resultado se assemelha ao encontrado no estudo realizado por Owens et al. ${ }^{14}$, que há duas décadas indica esta relação. Apesar da elevada prevalência de episódios de parassonias, nenhum outro estudo foi encontrado, sobre a temática em estudo na população de adolescentes, o que limita, ao menos em parte, as ilações que possam ser realizadas na discussão dos resultados encontrados no presente estudo.

Entre os pontos fortes deste estudo, destaca-se: amostra representativa dos estudantes do ensino médio do município; realização de análises separadas do tipo de exposição a diferentes tipos de tela; utilização de indicadores de parassonias associados a diferentes tipos e pontos de corte da exposição à tela. As principais limitações foram: não foi diferenciado o tempo de exposição considerando dias de semana e finais de semana; horário em que o adolescente dorme e acorda.

\section{CONCLUSÕES}

Conclui-se que o tempo insuficiente de sono e a exposição excessiva ao tempo de tela principalmente a TV estão associados positivamente com a percepção negativa da qualidade do sono. Observou-se que a exposição excessiva a TV esteve associada positivamente com episódios de parassonias em adolescentes.

Todos os autores declararam não haver qualquer potencial conflito de interesses referente a este artigo.

CONTRIBUIÇÕES DOS AUTORES: Cada autor contribuiu individual e significativamente para o desenvolvimento do manuscrito. AOS (0000-0003-2975-0043)*, LMFTO $(0000-0002-79377358)^{*}$ e RMT (0000-0002-2713-8670)* contribuíram na concepção e desenho da pesquisa. MAMS (0000-0002-2734-8416)* e AOS realizaram a pesquisa bibliográfica. AOS, MAMS e RMT foram os principais colaboradores na redação do manuscrito. AOS e LMFTO realizaram a coleta de dados. RMT realizou a análise dos dados e LMFTO, AOS e MAMS fizeram a análise crítica. Todos os autores revisaram e aprovaram a versão final do manuscrito. *ORCID (Open Researcher and Contributor ID).

\section{REFERÊNCIAS}

1. Brand S, Kirov R. Sleep and its importance in adolescence and in common adolescent somatic and psychiatric conditions. Int J Gen Med. 2011;4:425-42.

2. Carskadon MA, Acebo C, Jenni OG. Regulation of adolescent sleep: implications for behavior. Ann NY Acad Sci. 2004;1021:276-91.

3. Colrain IM, Baker FC. Changes in sleep as a function of adolescent development. Neuropsychol Rev. 2011;21(1):5-21

4. Hoefelmann LP, Silva KS, Barbosa FVC, Silva JAD, Nahas MV. Behaviors associated to sleep among high school students: cross-sectional and prospective analysis. Rev Bras de Cineantropom Desempenho Hum. 2014;16(Suppl 1):68-78.

5. Matricciani LA, Olds TS, Blunden S, Rigney G, Williams MT. Never enough sleep: a brief history of sleep recommendations for children. Pediatrics. 2012;129(3):548-56.

6. Hale L, Guan S. Screen time and sleep among school-aged children and adolescents: a systematic literature review. Sleep Med Rev. 2015;21:50-8.

7. Hysing M, Pallesen S, Stormark KM, Jakobsen R, Lundervold AJ, Sivertsen B. Sleep and use of electronic devices in adolescence: results from a large population-based study. BMJ Open. 2015;5(1):e006748.

8. Johnson JG, Cohen P, Kasen S, First MB, Brook JS. Association between television viewing and sleep problems during adolescence and early adulthood. Arch Pediatr Adolesc Med. 2004;158(6):562-8.

9. Foti KE, Eaton DK, Lowry R, McKnight-Ely LR. Sufficient sleep, physical activity, and sedentary behaviors. Am J Prev Med. 2011:41(6):596-602

10. Nuutinen T, Roos E, Ray C, Villberg J,Välimaa R, Rasmussen M, et al. Computer use, sleep duration and health symptoms: a cross-sectional study of 15-year olds in three countries. Int J Public Health. 2014;59(4):619-28.

11. Mesquita G, Reimão R. Nightly use of computer by adolescents: its effect on quality of sleep. Arq Neuropsiquiatr. 2007;65(2B):428-32

12. Yim WM, Cynthia STW, Donna WSH, Sil PL, Hei YT, Wing YY, et al. Association between screen viewing duration and sleep duration, sleep quality, and excessive daytime sleepiness among adolescents in Hong Kong. Int J Environ Res Public Health. 2014;11(11):11201-9.

13. Cajochen C, Frey S, Anders D, Späti J, Bues M, Pross A, et al. Evening exposure to a light-emitting diodes (LED)-backlit computer screen affects circadian physiology and cognitive performance. J Appl Physiol (1985). 2011;110(5):1432-8.

14. Owens J, Maxim R, McGuinn M, Nobile C, Msall M, Alario A. Television-viewing habits and sleep disturbance in school children. Pediatrics. 1999;104(3):e27.
15. American Academy of Sleep Medicine. International classification of sleep disorders, revised: Diagnostic and coding manual. Chicago, Illinois: American Academy of Sleep Medicine; 2005.

16. Bezerra J, Lopes AS, Hardmam CM, Tassitano RM, Tenório MCM, Barros MVG. Consumo de bebidas alcoólicas e tabagismo: associação com inatividade física no lazer e comportamento sedentário. Rev Andal Med Deporte. 2015:8:1-6.

17. Tenório MCM, Barros MD, Tassitano RM, Bezerra J, Tenório JM, Hallal PC. Atividade física e comportamento sedentário em adolescentes estudantes do ensino médio. Rev Bras Epidemiol. 2010;13(1):105-17.

18. Hoefelmann LP, Lopes Ada S, da Silva KS, Moritz P, Nahas MV. Sociodemographic factors associated with sleep quality and sleep duration in adolescents from Santa Catarina, Brazil: what changed between 2001 and 2011? Sleep Med. 2013;14(10):1017-23.

19. Bernardo MPS, Pereira EF, Louzada FM, Almeida V. Duração do sono em adolescentes de diferentes níveis socioeconômicos. J Bras Psiquiatr. 2009;58(4):231-7.

20. Dewald JF, Meijer AM, Oort FJ, Kerkhof GA, Bögels SM. The influence of sleep quality, sleep duration and sleepiness on school performance in children and adolescents: a meta-analytic review. Sleep Med. 2010;14(3):179-89.

21. Cain N, Gradisar M. Electronic media use and sleep in school-aged children and adolescents: a review. Sleep Med. 2010;11(8):735-42.

22. Instituto Brasileiro de Geografia e Estatística. Pesquisa nacional por amostra de domicílios. IBGE: Brasil; 2013 [acesso em 2015 nov 4]. Disponível em <http://www.ibge.gov.br/home/estatistica/populacao/ trabalhoerendimento/pnad2013/>

23. Mesquita G, Reimão R. Quality of sleep among university students: effects of nighttime computer and television use. Arq Neuropsiquiatr. 2010;68(5):720-5.

24. National Sleep Foundation. 2011 Sleep in America poll: communications technology in the bedroom 2011 [acesso em 2014 mai 18]. Disponível em <http://sleepfoundation.org/sites/default/files/sleepinamericapoll/SIAP_2011_Summary_of_Findings.pdf>

25. Sack RL, Auckley D, Auger RR, Carskadon MA, Wright KP Jr, Vitiello MV, Zhdanova IV; American Academy of Sleep Medicine. Circadian rhythm sleep disorders: part II, advanced sleep phase disorder, delayed sleep phase disorder, free-running disorder, and irregular sleep-wake rhythm. An American Academy of Sleep Medicine review. Sleep. 2007;30(11):1484-501.

26. Crowley SJ, Acebo C, Carskadon MA. Sleep, circadian rhythms, and delayed phase in adolescence. Sleep Med. 2007;8(6):602-12. 